H-mode access on JET and implications for ITER

This article has been downloaded from IOPscience. Please scroll down to see the full text article.

2008 Plasma Phys. Control. Fusion 50124053

(http://iopscience.iop.org/0741-3335/50/12/124053)

View the table of contents for this issue, or go to the journal homepage for more

Download details:

IP Address: 128.178.125.43

The article was downloaded on 22/06/2010 at 07:54

Please note that terms and conditions apply. 


\title{
H-mode access on JET and implications for ITER
}

\author{
Y Andrew ${ }^{1}$, T M Biewer ${ }^{2}$, K Crombe $^{3}$, D Keeling ${ }^{1}$, E de la Luna ${ }^{4}$, \\ C Giroud $^{1}$, N C Hawkes ${ }^{1}$, M Kempenaars ${ }^{1}$, A Korotkov ${ }^{1}$, A Meigs ${ }^{1}$,

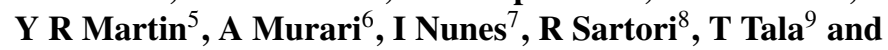 \\ JET EFDA contributors ${ }^{10,11}$ \\ ${ }^{1}$ Euratom/UKAEA Fusion Association, Culham Science Centre, Abingdon, OX14 3DB, UK \\ 2 Oak Ridge National Laboratory, Oak Ridge, Tennessee, TN 37831, USA \\ ${ }^{3}$ Department of Applied Physics, Ghent University, Belgium \\ ${ }^{4}$ Asociason EURATOM-CIEMAT para Fusion, CEIMAT, Madrid, Spain \\ ${ }^{5}$ Ecole Polytechnique Federal de Lausanne (EPFL), Centre de Recherche en Physique des \\ Plasmas, Association Euratom-Confederation Suisse, CH-1015 Lausanne, Switzerland \\ ${ }^{6}$ Consorzio RFX, ENEA-EURATOM Association, Padua, Italy \\ ${ }^{7}$ Associacao EURATOM/IST, Centro de Fusao Nuclear, Lisbon, Portugal \\ ${ }^{8}$ EFDA CSU, Boltzmannstrasse 2, 85748 Garching, Germany \\ ${ }^{9}$ Association EURATOM-Tekes, VTT, PO Box 1000, FIN-02044 VTT, Finland \\ 10 JET-EFDA, Culham Science Centre, Abingdon, OX14 3DB, UK \\ E-mail: yasmin.andrew@jet.uk
}

Received 6 June 2008, in final form 28 August 2008

Published 6 November 2008

Online at stacks.iop.org/PPCF/50/124053

\begin{abstract}
One of the critical issues for ITER is access to an H-mode regime with good confinement, $\mathrm{H}_{98}=1$. The most basic scaling laws for power threshold for the $\mathrm{L}-\mathrm{H}$ transition, $P_{\text {th }}$, take the variation with plasma density, magnetic field and plasma size into account. However, the large variations in the $P_{\text {th }}$ data from the values estimated with such simple scaling laws indicate other underlying dependencies. Another important consideration for ITER is that H-modes with higher values of energy confinement factors are often obtained with input power values much greater than $P_{\text {th }}$. This paper presents results from recent studies on JET to assess possible hidden variables for $\mathrm{H}$-mode access over a wide range of plasma conditions. Experimental results demonstrate that sensitivity to the magnetic shaping and divertor geometry could account for some of the scatter in the international power threshold database. Hysteresis in the $\mathrm{L}-\mathrm{H}$ transition $P_{\text {th }}$ has been studied in detail for the first time on JET by comparing values of $P_{\text {th }}$ at the forward and back H-mode transitions over a range of densities. The impact of the edge plasma rotation on $\mathrm{H}$-mode access has also been considered on JET with a toroidal field ripple scan across the $\mathrm{L}-\mathrm{H}$ and $\mathrm{H}-\mathrm{L}$ transitions. Finally, the total input power required relative to the measured value of $P_{\text {th }}$
\end{abstract}

\footnotetext{
11 See annex of Watkins et al 2006 Overview of JET Results Proc. 21st IAEA Fusion Energy Conf. (Chengdu, China).
} 
for access to a steady-state $\mathrm{H}$-mode with $\mathrm{H}_{98}=1$ has been examined for a highly shaped magnetic configuration. The implications of these results for the attainment of H-mode with good confinement on ITER are discussed.

(Some figures in this article are in colour only in the electronic version)

\section{Introduction}

Access to a good quality H-mode remains a crucial area of research on present-day tokamaks, especially with regard to extrapolation from current operating scenarios to ITER [1]. The foreseen method of access to steady-state H-mode on ITER is at low density, followed by an increase in density and power while remaining in H-mode to the required operating conditions [1]. Such a scenario is sensitive to the plasma density dependence of $P_{\text {th }}$, the level of hysteresis in the H-mode power and the power requirements above $P_{\text {th }}$ to reach $\mathrm{H}$-mode conditions with good confinement or $\mathrm{H}_{98}=1$. An improved understanding of the $\mathrm{L}-\mathrm{H}$ transition power dependences on present-day machines therefore has a direct impact on the optimization of conditions for H-mode access and maintenance on ITER.

Results are presented from recent experiments to further explore H-mode access in JET plasmas. In the following section the effect of the variation of magnetic configuration on $P_{\text {th }}$ and pedestal parameters is described. Section 3 provides a description of recent studies on the $\mathrm{H}-\mathrm{L}$ transition and the level of hysteresis in the H-mode power threshold on JET. Results from experiments to examine the influence of edge plasma, toroidal rotation velocity on the $\mathrm{L}-\mathrm{H}$ transition through changes to the level of toroidal field (TF) ripple are presented in section 4. The power requirements for access to $\mathrm{H}$-modes with $\mathrm{H}_{98}=1$ on JET are presented and discussed in section 5 and the paper concludes with a summary of the main results from these recent JET studies and their impact for H-mode access on ITER.

\section{Variation of the $\boldsymbol{P}_{\text {th }}$ density dependence}

A series of $\mathrm{L}-\mathrm{H}$ transition experiments have been run on the JET tokamak with three different magnetic configurations shown in figures $1(a)-(c)$. The aim of this study was to explore the effect of magnetic shaping and divertor configuration on the power threshold at the transition to and from the H-mode. A series of density scans were performed at fixed L-mode edge plasma electron density, using feedback control. All the shots shown in this paper had a lower single null magnetic configuration with the ion $\nabla B$ drift towards the X-point. The additional plasma heating was slowly ramped up and then back down at a rate of $1 \mathrm{MW} \mathrm{s}^{-1}$, using cocurrent neutral beam injection (NBI). In addition these shots had an input power of $1 \mathrm{MW}$ of ion cyclotron resonance heating (ICRH). The threshold power for the transitions in this study is defined as

$$
P_{\mathrm{th}}=P_{\text {in }}-\mathrm{d} W_{\text {dia }} / \mathrm{d} t,
$$

where $P_{\text {th }}$ is the power threshold, $P_{\text {in }}$ is the total input power and $\mathrm{d} W_{\text {dia }} / \mathrm{d} t$ is the rate of change in plasma energy. The $\mathrm{d} W_{\mathrm{dia}} / \mathrm{d} t$ is typically less than $15 \%$ on JET. Earlier experiments have shown the upper triangularity to have no influence on the power requirements for the $\mathrm{L}-\mathrm{H}$ transition [2]. This study also demonstrated that the increase in $\delta_{\text {lower }}$ from 0.23 to 0.33 reduced $P_{\text {th }}$ by up to $25 \%$. Since the $P_{\text {th }}$ is known to be very sensitive to divertor geometry [2], the reduction in $P_{\text {th }}$ with increased $\delta_{\text {lower }}$ could be attributed to the lowering of the X-point height 

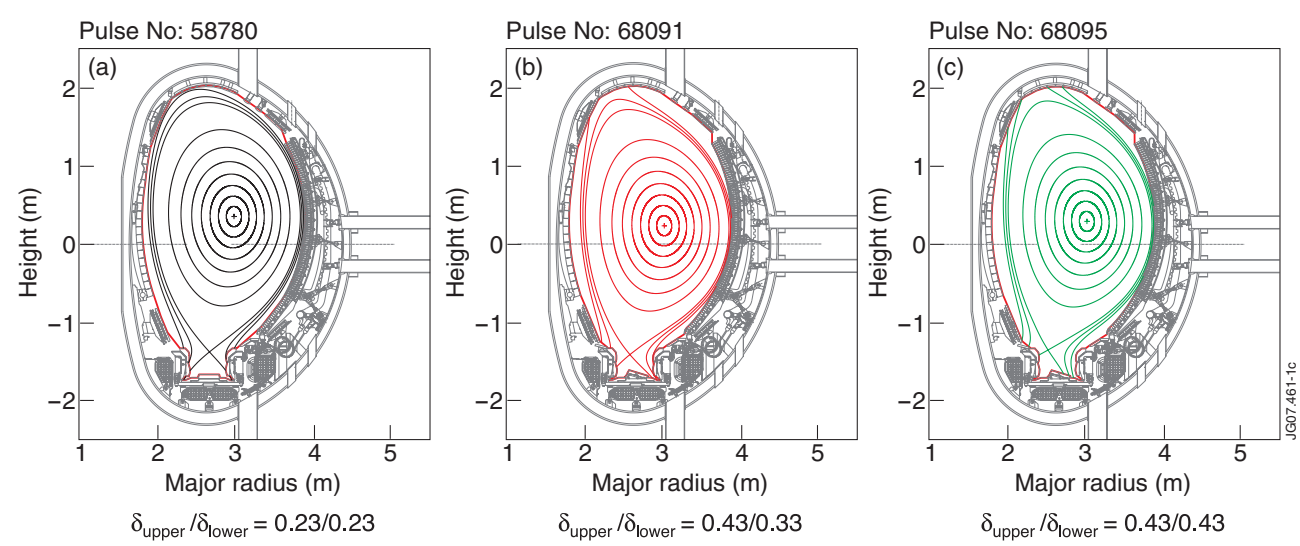

Figure 1. Magnetic configurations used in density scans at $2.5 \mathrm{MA} / 2.7 \mathrm{~T}$.

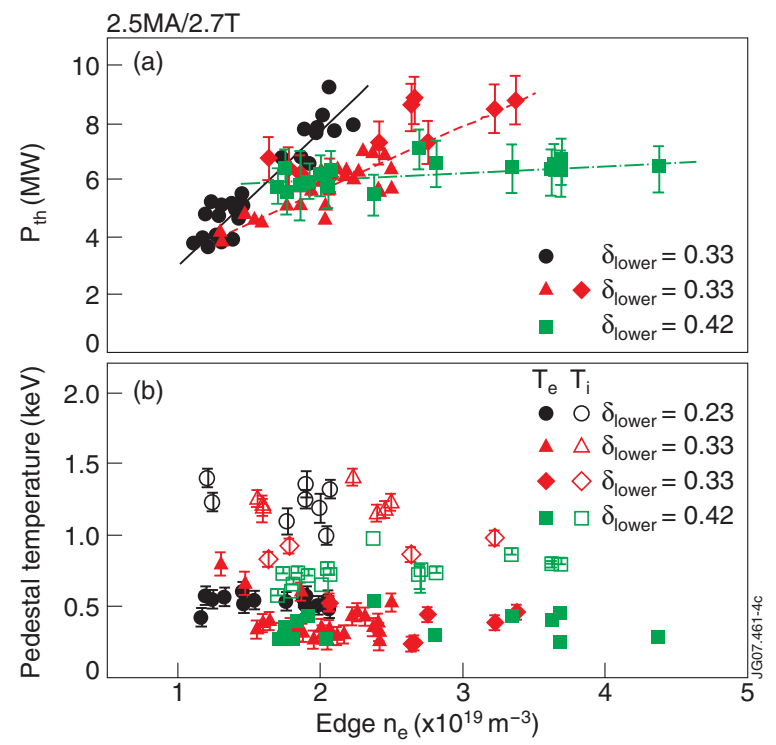

Figure 2. Comparison of the (a) $P_{\mathrm{th}}$ values and $(b)$ pedestal top $T_{\mathrm{i}}$ and $T_{\mathrm{e}}$ for plasmas with $\delta_{\text {upper }} / \delta_{\text {lower }}=0.23 / 0.23,0.43 / 0.33$ and $0.43 / 0.43$.

by $6 \mathrm{~cm}$ along with the movement of the outer strike point from the vertical to the horizontal target plate. The mechanisms by which changes in the X-point height or strike point position influence the $\mathrm{L}-\mathrm{H}$ transition are not yet fully understood.

The $P_{\text {th }}$ and pedestal temperature at the L-H transition are plotted as a function of line average, edge plasma electron density, $\bar{n}_{\mathrm{e}}$, for all three configurations in figures $2(a)$ and $(b)$, respectively. The pedestal ion temperatures have been measured with the edge charge exchange recombination spectroscopy diagnostic [3] while the pedestal electron temperatures have been measured with a multichannel electron cyclotron emission radiometer [4]. The line integrated edge $n_{\mathrm{e}}$ is measured with an interferometer [5] along a single chord. The line average edge $\bar{n}_{\mathrm{e}}$ is calculated by dividing the measured edge $n_{\mathrm{e}}$ by the chord length in the plasma. Two sets of points are shown in figure 2 at $\delta=0.33$ (diamonds and triangles) corresponding to two 
different JET divertor target plates. The results clearly show the density dependence of $P_{\text {th }}$ to be weaker with increased lower triangularity or more likely as a result of changes in the divertor geometry. An unconstrained fit to $P_{\text {th }}$ for each data set gives a dependence of

$$
\begin{aligned}
P_{\text {th }} & \propto \bar{n}_{\mathrm{e}}^{0.12( \pm 0.04)}, & & \text { for } \delta_{\text {upper }} / \delta_{\text {lower }}=0.43 / 0.43, \\
P_{\text {th }} & \propto \bar{n}_{\mathrm{e}}^{0.82( \pm 0.07)}, & & \text { for } \delta_{\text {upper }} / \delta_{\text {lower }}=0.43 / 0.33, \\
P_{\text {th }} & \propto \bar{n}_{\mathrm{e}}^{1.26( \pm 0.09)}, & & \text { for } \delta_{\text {upper }} / \delta_{\text {lower }}=0.23 / 0.23 .
\end{aligned}
$$

The strong dependence of $P_{\text {th }}$ on the triangularity and divertor geometry could be an important issue for ITER in terms of $\mathrm{H}$-mode sustainment following the $\mathrm{L}-\mathrm{H}$ transition, with operational requirements for increases in $\mathrm{H}$-mode plasma density directly following the L$\mathrm{H}$ transition under conditions of limited auxiliary power. Pedestal $T_{\mathrm{i}}$ and $T_{\mathrm{e}}$ are plotted in figure 2(b) for the corresponding $P_{\text {th }}$ data shown in figure 2(a). It can be seen that the pedestal $T_{\mathrm{i}}$ is consistently higher than the pedestal $T_{\mathrm{e}}$ at the $\mathrm{L}-\mathrm{H}$ transition across the density scan. In addition, the highest triangularity shape configuration has the lowest pedestal temperatures, with mean values of $T_{\mathrm{i}}=735( \pm 100) \mathrm{eV}$ and $T_{\mathrm{e}}=353( \pm 88) \mathrm{eV}$ for the highest triangularity plasmas and $T_{\mathrm{i}}=1129( \pm 171) \mathrm{eV}$ and $T_{\mathrm{e}}=396( \pm 138) \mathrm{eV}$ for $\delta_{\text {upper }} / \delta_{\text {lower }}=0.43 / 0.33$. These results provide further indication that the pedestal temperature is not the controlling parameter for the $\mathrm{L}-\mathrm{H}$ transition.

\section{H-L transition studies}

Some of the shots in the density scans with $\delta_{\text {upper }} / \delta_{\text {lower }}=0.43 / 0.33$ and $0.43 / 0.43$, described in section 2 and shown in figure 2, were also carried out with a slow power ramp-down to study the $\mathrm{H}-\mathrm{L}$ transition behaviour over a range of densities. The $\mathrm{L}-\mathrm{H}$ and $\mathrm{H}-\mathrm{L}$ transition $P_{\text {th }}$, $T_{\mathrm{i}}$ and $T_{\mathrm{e}}$ are compared in figure 3 for a subset of points shown in figure 2. The $P_{\text {th }}$ data for the $\mathrm{L}-\mathrm{H}$ and $\mathrm{H}-\mathrm{L}$ transitions are very similar and the data show no evidence of hysteresis in the threshold power for either configuration across the density range covered. The pedestal $T_{\mathrm{e}}$ is also very similar for the forward and back transitions into and out of the H-mode, for both magnetic configurations. These $P_{\text {th }}$ measurements suggest that it may not be possible to rely on hysteresis in the H-mode power threshold in order to access the high density, high confinement $\mathrm{H}$-mode operating regime with input power less than $P_{\text {th }}$ at a given plasma density on ITER.

\section{TF ripple}

The finite number of TF coils on tokamaks results in the toroidal variation of the magnetic field, TF ripple or $\delta$. The TF ripple is expected to be around $\delta=0.5 \%$ at the outer separatrix on ITER [6] and it is known that TF ripple ion losses can lead to significant counter toroidal rotation velocity, $v_{\phi}$, at the plasma edge [7]. Any variation in $v_{\phi}$ or the poloidal rotation velocity, $v_{\theta}$, can strongly influence the edge radial electric field, $E_{r}$, which is in turn thought to play a significant role in turbulence suppression. JET has the capability of varying the TF ripple amplitude from a standard value of $\delta=0.08 \%$ up to a maximum of $\delta=3 \%$ and is therefore in a unique position to study the effect of TF ripple amplitude on the $\mathrm{L}-\mathrm{H}$ and $\mathrm{H}-\mathrm{L}$ transitions.

A series of shots were run with $I_{\mathrm{p}} / B_{\mathrm{t}}=2.0 \mathrm{MA} / 2.2 \mathrm{~T}$ with varying levels of L-mode target electron density, $\bar{n}_{\mathrm{e}}$, controlled using active feedback. Two different amplitudes of TF ripple were used, $\delta \simeq 0.08 \%$ and $1.1 \%$ at the outer separatrix, with a low triangularity magnetic configuration of 0.2 . The threshold power, $P_{\mathrm{th}}$, has been corrected for fast ion power losses 

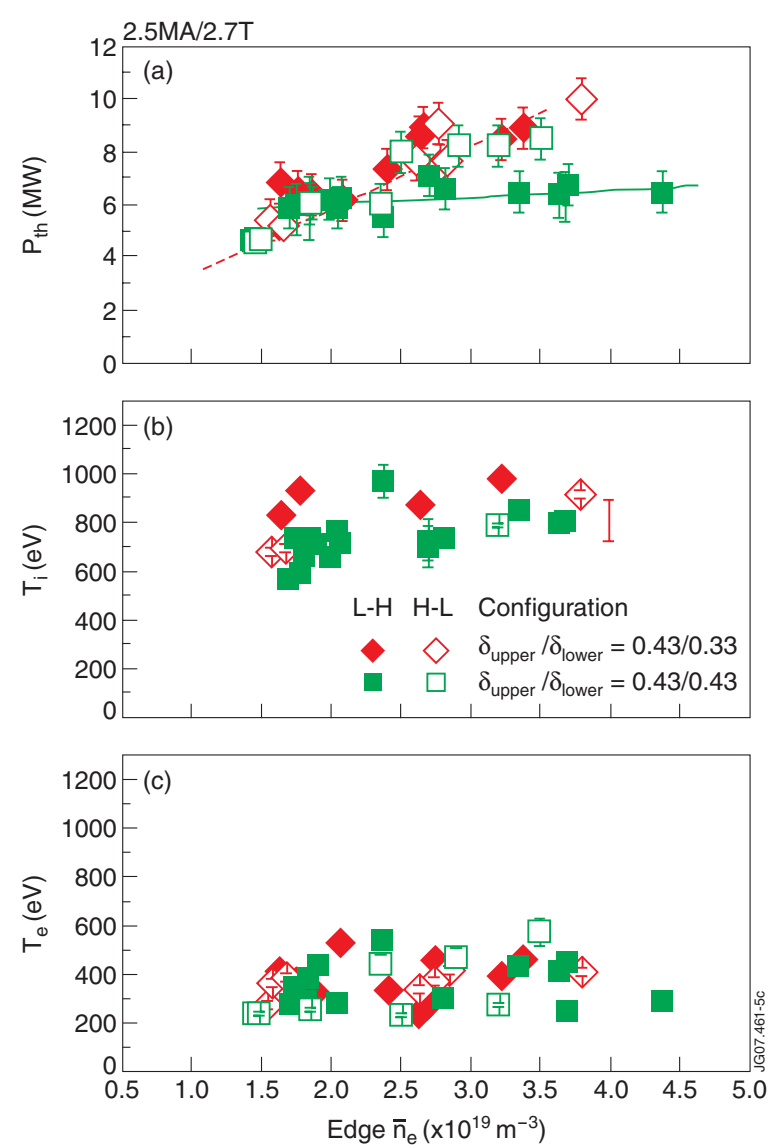

Figure 3. Comparison of the $\mathrm{L}-\mathrm{H}$ and $\mathrm{H}-\mathrm{L}(a) P_{\mathrm{th}},(b)$ pedestal $T_{\mathrm{i}}$ and $(c)$ pedestal $T_{\mathrm{e}}$ for plasmas with $\delta_{\text {upper }} / \delta_{\text {lower }}=0.43 / 0.33$ and $0.43 / 0.43$.

due to TF ripple, $P_{\mathrm{CORR}}$. Values of $P_{\mathrm{CORR}}$, pedestal $T_{\mathrm{i}}$ and pedestal $T_{\mathrm{e}}$ are plotted in figure $4(i)$ as a function of edge $\bar{n}_{\mathrm{e}}$ for the $\mathrm{L}-\mathrm{H}$ and $\mathrm{H}-\mathrm{L}$ transitions. The data show the power threshold and pedestal temperatures to be unaffected by the level of TF ripple. The corresponding values of $v_{\phi}$ and $v_{\theta}$ measured at the location of the pedestal $T_{\mathrm{i}}$ (at $\rho \simeq 0.95$ ) and also further within the confined plasma at $\rho=0.85$ are plotted in figure 4(ii). The edge $v_{\theta}$ was observed to be unaffected by the level of TF ripple in these shots. Plasmas with $\delta=0.08 \%$ TF ripple were characterized by $v_{\phi}=-3$ to $-34 \mathrm{~km} \mathrm{~s}^{-1}$ in the co-current direction across the edge region both before and following the transition to and from H-mode. In contrast, plasmas with increased TF ripple, $\delta=1.1 \%$, were observed to counter rotate across the edge region both before and after the $\mathrm{L}-\mathrm{H}$ transition, with values ranging from $v_{\phi}=+6 \mathrm{~km} \mathrm{~s}^{-1}$ to $+19 \mathrm{~km} \mathrm{~s}^{-1}$. Therefore, large changes in the direction of toroidal rotation velocity across the pedestal region do not appear to make a significant difference to the power requirements for the $\mathrm{L}-\mathrm{H}$ transition on JET.

It is interesting to note that for the last $2 \mathrm{~s}$ of the power ramp-down, the level of edge $\bar{n}_{\mathrm{e}}$ falls due to reduced gas puffing and the edge plasma $v_{\phi}$ decreased dramatically and changed direction from counter- to co-current for the shots with $1.1 \% \mathrm{TF}$ ripple. The edge $v_{\phi}$ remained in the co-current direction for these shots for $1 \mathrm{~s}$ before and during the $\mathrm{H}-\mathrm{L}$ transitions, as 

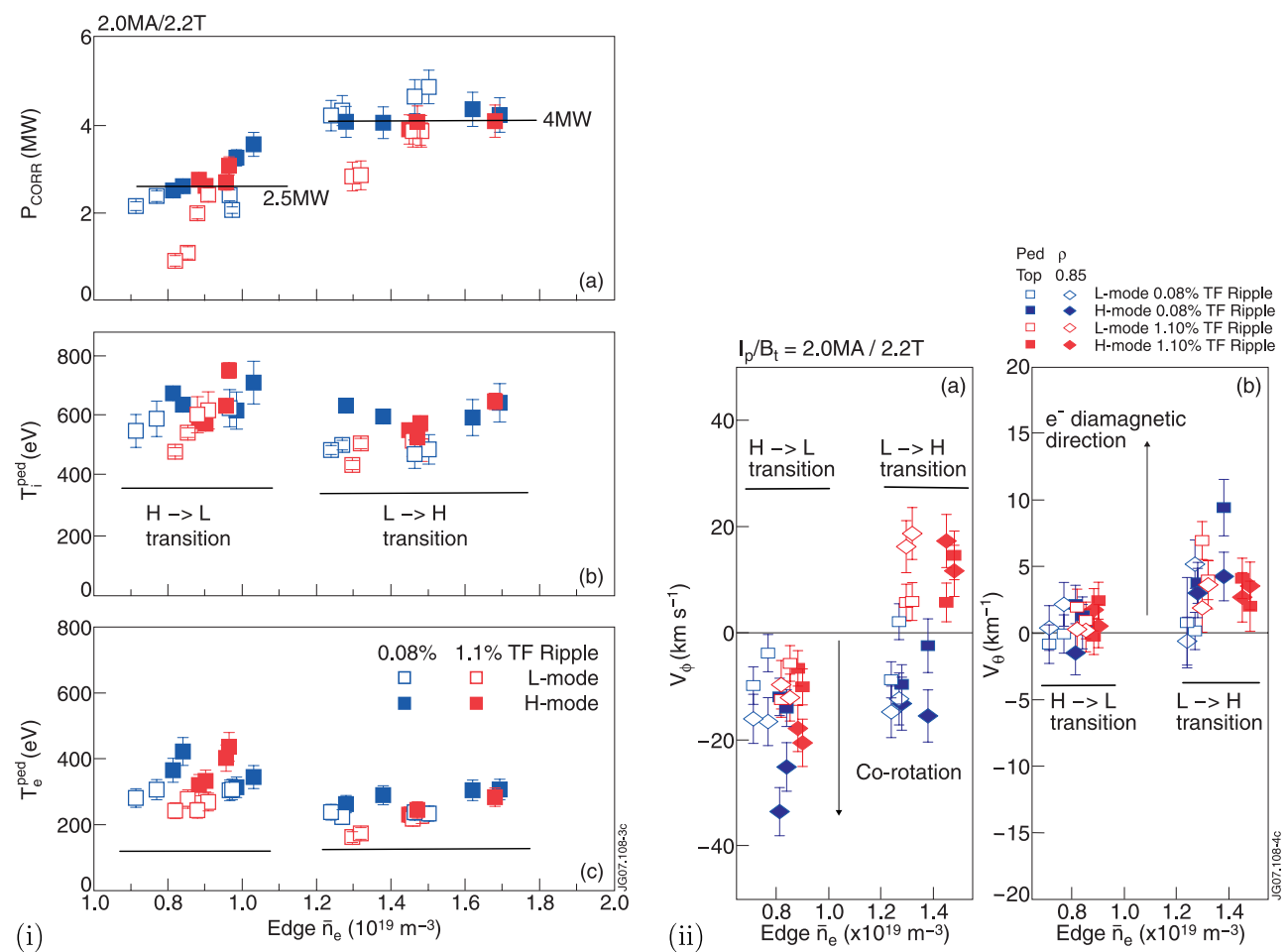

Figure 4. ( $i$ ) Values of (a) PCORR $(b)$ pedestal $T_{\mathrm{i}}$ and $(c)$ pedestal $T_{\mathrm{e}}$ at $\mathrm{L}-\mathrm{H}$ transition as a function of edge $\bar{n}_{\mathrm{e}}$ for shots with and without TF ripple across the L-H and $\mathrm{H}-\mathrm{L}$ transitions. (ii) (a) $v_{\phi}$ and (b) $v_{\theta}$ plotted as a function of edge $\bar{n}_{\mathrm{e}}$ for shots with and without TF ripple at the location of top of the $T_{\mathrm{i}}$ pedestal and at $\rho=0.85$ across the $\mathrm{L}-\mathrm{H}$ and $\mathrm{H}-\mathrm{L}$ transitions.

shown in figure 4(ii). This result demonstrates that low density, low power conditions exist under which the application of significant TF ripple does not provide sufficient counter-torque for edge plasma counter-rotation.

\section{H-mode power requirements for good confinement}

The minimum input power necessary for access to the $\mathrm{H}$-mode with $\mathrm{H}_{98}=1$ relative to the experimentally measured L-H transition $P_{\text {th }}$ has been studied by running a series of shots with constant input power. The input power was varied in successive plasmas to obtain a steadystate, fine power scan for the $\delta_{\text {upper }} / \delta_{\text {lower }}=0.43 / 0.44$ configuration. As shown in figure $5(i)$, at the lowest level of input power, $P_{\text {in }}=8.6 \mathrm{MW}$, the plasma remained in a state of transition between ELM-free H-mode and high frequency, irregular Type-III ELMs, The average H-mode confinement factor over the $1 \mathrm{~s}$ time window, from 23 to $24 \mathrm{~s}$, for this shot was $\mathrm{H}_{98}=0.8$ as shown in figure 4(ii). Following an increase in the total input power to $P_{\text {in }}=9.3 \mathrm{MW}$, the plasma remained in a mixed Type-III ELMs/ELM-free state seen in figure 5( $i$ ) for shot 68218 . A steady-state Type-I ELMy H-mode was subsequently accessed in shot 68220 with a total input power of $P_{\text {in }}=9.7 \mathrm{MW}$ with an ELM frequency of $f_{\mathrm{ELM}}=27 \mathrm{~Hz}$ and $\mathrm{H}_{98}=0.9$, also plotted in figure $4(\mathrm{ii})$. A further increase in $P_{\text {in }}$ to $10.5 \mathrm{MW}$ led to a transient, mixed Type-I ELMy/ELM-free phase $\mathrm{H}$-mode with $\mathrm{H}_{98}=0.9$. In order to extend the power scan, shot 68216 was run with $P_{\text {in }}=15.4 \mathrm{MW}$ and the plasma remained in a steady Type-I ELMy H-mode with 

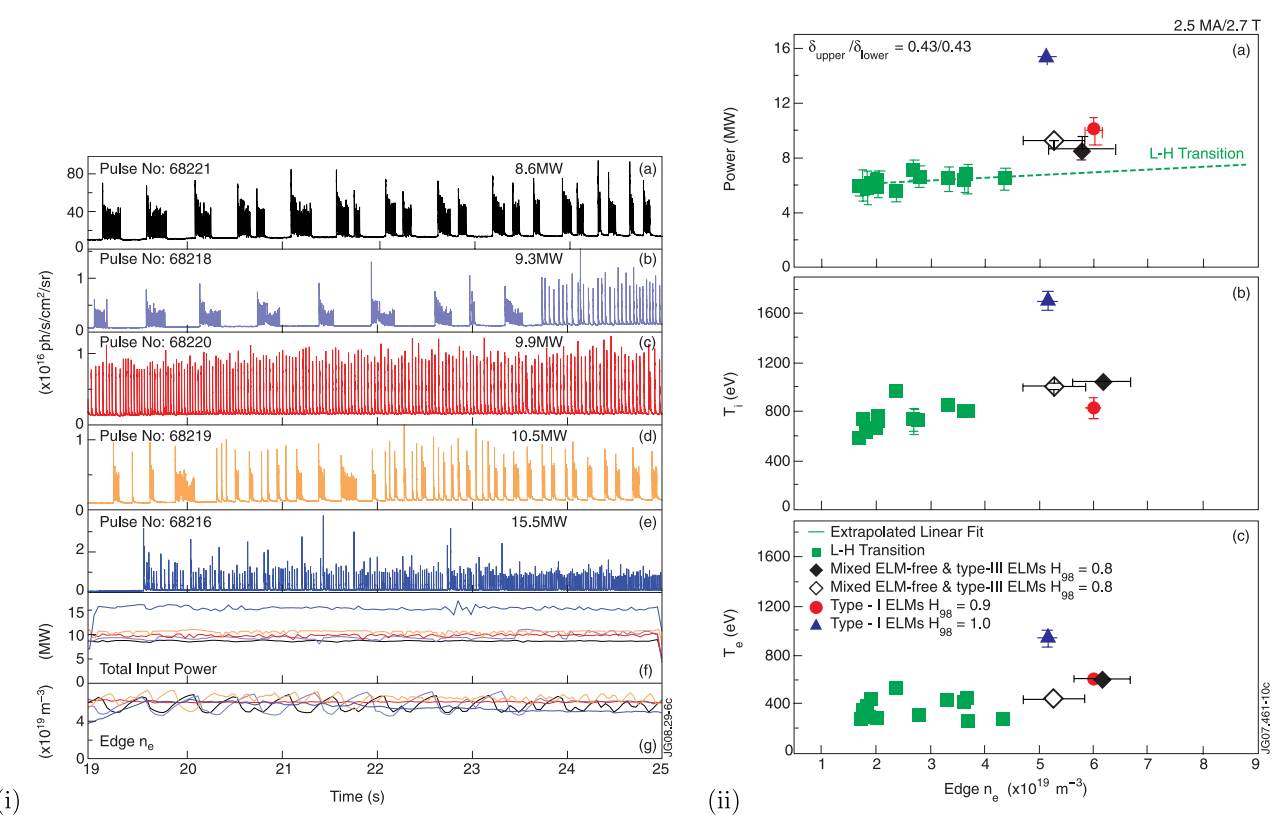

Figure 5. (i) (a)-(e) Variation of ELM frequency and $\mathrm{D}_{\alpha}$ characteristics with increasing levels of $(f)$ steady-state input power and $(g)$ for similar edge plasma density. (ii) Comparison of $(a) P_{\text {th }}$, (b) $T_{\mathrm{i}}$ and $(c) T_{\mathrm{e}}$ at the L-H transition with power levels for mixed ELM-free and Type-III ELM phases and for steady-state Type-I ELMs.

$f_{\mathrm{ELM}}=60 \mathrm{~Hz}$ and $\mathrm{H}_{98}=1.0$, as plotted in figures $5(i)$ and $(i i)$. These results demonstrate that $P_{\text {in }}=1.5 P_{\text {th }}$ for Type-I ELM access with $\mathrm{H}_{98}=0.9$ and $P_{\text {in }}=2.2 P_{\text {th }}$ for access to a Type-I ELMy regime with $\mathrm{H}_{98}=1.0$ on JET for this higher triangularity configuration. These findings are in broad agreement with the results $P_{\text {in }}=2 P_{\text {th }}$ reported by Sartori et al in [8] and confirm that it may not be possible to access an H-mode with good confinement without input power significantly above the L-H transition $P_{\text {th }}$ at a given plasma density on ITER.

\section{Conclusions}

Results have been presented from recent experiments to study H-mode access on JET and demonstrate the edge plasma density dependence of $P_{\text {th }}$ for H-mode access to vary significantly with magnetic configuration. One of the contributing factors may be the variation in the $\mathrm{X}$-point proximity to the divertor floor and strike point location required to accommodate the differences in lower triangularity. The sensitivity of the $\mathrm{L}-\mathrm{H}$ transition $P_{\mathrm{th}}$ and its density dependence to magnetic shaping and divertor geometry could be an important consideration for $\mathrm{H}$-mode access power requirements on ITER. A simple power threshold scaling law, with a single plasma density dependence, does not describe these results and further work is needed for a theoretical understanding of these effects.

No experimental evidence for hysteresis in the power threshold for the L-H transition was found on JET. Therefore, any operation scenarios on ITER that rely on hysteresis in power for access to a high density $\mathrm{H}$-mode following the $\mathrm{L}-\mathrm{H}$ transition with limited additional heating should be considered very carefully.

Density scans performed with and without significant levels of TF ripple on JET show no change in $P_{\text {th }}$ for H-mode access despite large differences in the edge toroidal rotation velocity 
direction and magnitude. A level of TF ripple of $\delta=0.5 \%$ therefore may not have a large impact on the power requirements for the L-H transition on ITER.

Finally, at input powers close to the $P_{\mathrm{th}}$, JET H-modes typically have $\mathrm{H}$-mode confinement factor values of around $\mathrm{H}_{98}=0.8$. Total input powers of $P_{\text {in }}>1.5 P_{\text {th }}$, were found to be necessary for steady-state $\mathrm{H}$-mode access with $\mathrm{H}_{98}=1$ and Type-I ELMs on JET. These results suggest that auxiliary power with a significant margin above $P_{\text {th }}$ would be necessary on ITER for detailed H-mode studies in hydrogen, helium or deuterium plasmas.

\section{Acknowledgments}

This work was carried out within the framework of the European Fusion Development Agreement and was partly funded by the United Kingdom Engineering and Physical Sciences Research Council and by the European Communities under the contract of Association between EURATOM/UKAEA. The views and opinions expressed therein do not necessarily reflect those of the European Commission.

(C) Euratom 2008.

\section{References}

[1] Doyle E J et al 2007 Nucl. Fusion 47 S18-127

[2] Andrew Y et al 2004 Plasma Phys. Control. Fusion 46 A87-93

[3] Andrew Y et al 2006 Rev. Sci. Instrum. 77 10E913

[4] de la Luna E et al 2003 Rev. Sci. Instrum. 74 1414-20

[5] Murari A et al 2006 Rev. Sci. Instrum. 77073505

[6] Saibene G et al 2007 Nucl. Fusion 47 969-83

[7] Urano H et al 2006 Plasma Phys. Control. Fusion 46 A193-9

[8] Sartori R et al 2004 Plasma Phys. Control. Fusion 46 723-50 